\title{
Essais
}

ESSAIS

Revue interdisciplinaire d'Humanités

\section{Le renouvellement des élites et le marché de la presse à l'époque napoléonienne}

\section{Iwan-Michelangelo D'Aprile}

Traducteur : Mathilde Lerenard

\section{OpenEdition}

\section{Journals}

Édition électronique

URL : http://journals.openedition.org/essais/8107

DOI : 10.4000/essais. 8107

ISSN : 2276-0970

\section{Éditeur}

École doctorale Montaigne Humanités

\section{Édition imprimée}

Date de publication : 15 juillet 2014

Pagination : $42-54$

ISBN : 978-2-9544269-3-8

ISSN : 2417-4211

\section{Référence électronique}

Iwan-Michelangelo D'Aprile, « Le renouvellement des élites et le marché de la presse à l'époque napoléonienne », Essais [En ligne], 5 | 2014, mis en ligne le 13 avril 2021, consulté le 15 avril 2021. URL : http://journals.openedition.org/essais/8107 ; DOI : https://doi.org/10.4000/essais.8107 


\title{
Le renouvellement des élites et le marché de la presse à l'époque napoléonienne
}

\author{
Iwan-Michelangelo D'Aprile
}

Les révolutions et les grands bouleversements sociaux se caractérisent toujours par un renouvellement des élites, au cours duquel les couches de population marginales gagnent en influence politique et sociale et de nouvelles perspectives de carrières s'ouvrent à elles. Ainsi, une transformation profonde des élites sociales s'opère avec la Révolution française et l'époque napoléonienne, qui fait l'objet depuis un certain temps de recherches historiques ${ }^{1}$, par exemple sur l'armée et l'élite administrative. Dans les principautés allemandes, la situation se révèle bien plus opaque. Même dans les États qui faisaient partie du système de puissances napoléonien, on n'observe pas cette mutation de l'ancienne société d'ordres, ni cette ascension de nouvelles catégories sociales qui se produisent alors en France.

S’il est un domaine où le défi révolutionnaire et napoléonien a conduit indéniablement à de nouvelles perspectives de carrière chez des couches sociales jusqu'alors sous-privilégiées, il s'agit du marché de publications sur l'histoire politique contemporaine, en pleine transformation, et de la presse politique étatique de plus en plus active ${ }^{2}$. Suivant l'exemple de Napoléon, les gouvernements de tous les grands États reconnurent la nécessité de soigner les relations publiques et tentèrent de faire pencher l'opinion dans leur sens ${ }^{3}$. Ainsi, la possibilité de vivre de l'écriture se présenta à un nombre croissant d'auteurs.

À la suite du journalisme politique des Lumières tardives (la Spätaufklärung) chez des auteurs comme Wilhelm Ludwig Wekhrlin, Peter Adolph Winkopp, Schubarth, August Ludwig Schlözer ou Christoph Martin Wieland

1 Werner Giesselmann, Die brumairianische Elite, Stuttgart, 1977 ; Walter Markov, Napoleon und seine Zeit. Geschichte und Kultur des Grand Empire, Leipzig, 1996.

2 Darnton Robert, The literary underground oft he Old Regime, Cambridge, Mass, 1982.

3 Wolfgang Piereth, Propaganda im 19. Jahrhundert. Die Anfänge staatlicher Politik in Deutschland (1800-1817), in Ute Daniel u. Wolfram Siemann (Hg.), Propaganda, Meinungskampf, Verführung und Sinnstiftung (1789-1989), Frankfurt am M., 1994, p. 21-43. 
- qui furent l'objet d'une analyse approfondie par Jean Mondot et Tristan Coignard ${ }^{4}$ - une nouvelle forme d'historiographie contemporaine se constitue. Celle-ci s'exprime à travers des formes populaires comme les périodiques historiques, les livres de poche et les almanachs. Elle est, comme l'a constaté Reinhart Koslleck, dès le début "repoussée dans la sphère journalistique, dans la rédaction de quotidiens $"^{5}$. Dès 1770 déjà, on remarque une augmentation sensible de la création de journaux historiques et politiques qui ne décroît qu'à partir de 1830 environ. Avec les bouleversements politiques et économiques et les formes de publicité qui se transforment dans le cadre de la Révolution française, ce phénomène s'accélère et cela conduit en particulier à la création de nouveaux journaux traitant de l'histoire contemporaine, dont le programme est souvent visible à travers leur titre, comme l'Histoire de l'époque actuelle (Geschichte der gegenwärtigen Zeit. Strasbourg, 1790-1793), Magazine raisonné de l'essentiel de l'histoire contemporaine (Räsonnierendes Magazin des Wichtigsten an der Zeitgeschichte. Salzbourg, 1791-1792) ou Klio. Un mensuel pour l'histoire française contemporaine (Klio. Eine Monatsschrift für die französische Zeitgeschichte. Leipzig, 1795-1796).

Dans l'espace nord-allemand, on peut citer le journal d'histoire contemporaine le plus lu, Minerva, de Johann Wilhelm Archenholz, mais aussi les Préliminaires de la paix (Friedenspräliminärien) d'Ernst Ludwig Huber, créées dans le cadre de la décennie de paix qui suivit le traité de Bâle, ou encore les journaux de Johann Friedrich Reichhardt aux titres caractéristiques France et Allemagne. Les journaux d'histoire contemporaine les plus ambitieux au niveau conceptuel autour de 1800 sont d'une part le Journal historique (Historisches Journal) de Friedrich Gentz (1799-1800) et le périodique Histoire et Politique (Geschichte und Politik) publié par Karl Ludwig Woltmann et conçu à la fois comme suite et comme opposition au journal de Gentz. Ces deux périodiques furent tous deux publiés à près de 2000 exemplaires.

Il s'agit pour la suite de présenter le renouvellement des élites dans la presse politique autour de 1800 à partir d'exemples et suivant trois étapes : premièrement, les nouvelles perspectives de carrière pour une nouvelle catégorie d'auteurs, les " journalistes-historiens ", seront présentées. Les réseaux européens et en particulier franco-allemands constitueront le deuxième axe d'étude. Enfin, les continuités dans la presse politique au-delà de l'époque

4 Mondot Jean, W.L. Wekhrlin. Un publiciste des lumières. 2 vol., Bordeaux, Presses Universitaires, 1986 ; Coignard Tristan, L'apologie du débat public. Réseaux journalistiques et pouvoirs dans l'Allemagne des Lumières, Bordeaux, 2009.

5 Koselleck Reinhart, Stetigkeit und Wandel aller Zeitgeschichten. Begriffsgeschichtliche Anmerkungen, in du même auteur, Zeitschichten. Studien zur Historik, Frankfurt am M., 2003, p. 246-264. L'indication de Koselleck selon laquelle celle-ci aussi avait " une telle ampleur " avec des auteurs comme Bruno Bauer, Karl Marx, Lorenz von Stein, Michelet ou Adolphe Thiers, " dont les écrits sur l'histoire contemporaine font encore partis aujourd'hui des lectures renouvelables ", est étudiée dans la suite de l'article à l'exemple de leurs prédécesseurs. 
napoléonienne dans les deux grands États que constituent la Bavière et la Prusse seront discutées à partir de deux exemples.

\section{Les historiens-journalistes}

Le marché de la presse donna pour la première fois la possibilité d'accéder à des revenus qui dépassaient les salaires de l'administration publique et permit à un nombre croissant de journalistes et d'éditeurs de vivre de la publication de périodiques politiques. Un nouveau type d'auteurs se forma ainsi : celui du journaliste-historien ou de l'« écrivain du temps ». Ces écrivains du temps n'étaient pas, pour la plupart, des diplômés de l'enseignement supérieur ou des universitaires, mais vivaient - en tant que "Privatpersonen mit public character", comme on les désigne à l'époque ${ }^{6}$ - d'écrits journalistiques ou de la publication de journaux historiques. Cela ne les empêchait aucunement de chercher à influencer les gouvernements, et si possible, de prendre un poste de conseiller politique et médiatique dans la fonction publique. La presse politique de l'État qui devient de plus en plus active après Napoléon présentait de nombreuses perspectives de carrière dans ce domaine.

$\mathrm{La}$ fourchette des revenus allait des journalistes journaliers et auteurs prolifiques aux gros salaires et aux carrières européennes surprenantes de journaliste-historien, comme celles de Friedrich Gentz, Johannes Müller ou Heinrich Zschokke. Le gouvernement prussien fit par exemple venir Johannes Müller de Vienne en 1804, le nomma membre ordinaire de l'Académie des Sciences tout en lui offrant le titre de Conseiller secret et lui versa un revenu annuel de 3000 reichstalers. Plus tard, il devint ministre dans le royaume de Westphalie. Gentz parcourut le chemin inverse : il quitta en 1802 la Prusse et se fit employer en tant que Conseiller impérial avec un revenu annuel de 4000 florins. En plus de cela, il recevait 800 livres sterling par an du gouvernement anglais pour ses services diplomatiques. Le fils de tisserand originaire de Magdebourg et écrivain prolifique Heinrich Zschokke fut quant à lui promu chef du Bureau de la Culture Nationale de la république helvétique.

Des auteurs moins connus virent également des perspectives d'ascension sociale s'ouvrir à eux, comme l'auteur autrichien de récits de voyage, Joseph Widemann, qui devint un employé de presse important des gouvernements français et bavarois, ou encore le juif Karl Julius Lange qui effectua des années 1790 à 1805 le travail médiatique chez Hardenberg, et publia en 1806 le premier quotidien berlinois, Le Télégraphe, avant d'être nommé Conseiller à la cour d'Isenberg sous Napoléon. Un autre exemple de carrière journalistique impressionnante est celui de Friedrich Buchholz : il abandonna en 1800 son poste d'enseignant maigrement rémunéré à l'école militaire de Brandebourg sur l'Havel pour s'installer à Berlin en tant qu'écrivain. Après quelques romans

6 Anonyme : Kabinett Berlinischer Karaktere, 1808, p. IV f. 
et succès honorables grâce à des traités théoriques, Buchholz réussit, en tant que rédacteur du Vossische Zeitung chez Unger, dès les huit premiers mois, à faire monter les tirages à 1500 abonnés et à décupler les bénéfices. À la mort d'Unger et la reprise de la maison d'édition par sa veuve Friderike Unger, les livres de compte de la maison d'édition indiquent qu'il restait à verser la somme de 2000 reichstalers à Buchholz. ${ }^{7}$ À partir de 1805, ce dernier devint l'employé le plus important des Annales européennes (Europäische Annalen) de Cotta. Il connut ses plus grands succès de marché lors de sa coopération avec Johann Daniel Sander et avec son écrit intitulé Galerie de personnalités prussiennes (Galerie Preußischer Charaktere, 1808) qui fit scandale et dont la première édition à hauteur de 6000 exemplaires fut épuisée lorsque l'ouvrage fut interdit par l'organe de censure en France. Dans son autoportrait publié dans la Galerie de personnalités prussiennes, Buchholz indique fièrement qu'il est en mesure, depuis huit ans, de nourrir sa famille (qui compte six membres) simplement grâce à "son zèle d'écrivain " ${ }^{8}$. À l'époque, même ses opposants politiques comme Friedrich Gentz reconnaissent son "immense popularité "? et vont jusqu'à le désigner, au même titre qu'Adam Müller, comme « leader de la presse politique en Allemagne " ${ }^{10}$. À partir de 1811, Buchholz devint l'un des employés de presse politique les plus importants au sein de la chancellerie d'Hardenberg, de laquelle il influença considérablement l'opinion publique jusque dans les années 1830 avec son Mensuel historique et politique pour l'Allemagne (bistorisch-politische Monatsschrift für Deutschland).

Certains parvinrent même à gagner de l'argent en cessant d'écrire - c'est le cas d'Adam Müller qui reçut, durant la période de réformes du gouvernement prussien, une allocation d'attente dont le montant s'élevait à 1200 reichstalers, afin que celui-ci se taise, parce qu'il s'était fait le porte-parole de l'opposition venant de la noblesse traditionnelle ${ }^{11}$. Si on compare cela avec la moyenne des possibilités de revenus pour le travail intellectuel au sens large, qui vont de 100-150 rt par an pour les précepteurs à 300-500 rt pour les employés de la petite et moyenne administration, il n'est pas étonnant que de nombreux auteurs abandonnèrent leurs occupations pour se reconvertir totalement dans le journalisme politique. Garlieb Merkel par exemple, quitta son poste de

7 Lehmstedt Mark, "Ich bin nun vollends zur Kaufmannsfrau verdorben ». Au sujet du rôle de la femme dans l'histoire de l'édition d'après l'exemple de Friedericke Helene Unger (17511813), in Leipziger Jahrbuch zur Buchgeschichte, 6, 1996, p. 81-154, p. 105.

8 "In Berlin hatte er für seine Subsistenz keine andere Grundlage, als seinen schriftstellerischen Fleiß». Buchholz Friedrich, Galerie Preußischer Charaktere. Aus der Französischen Handschrift übersetzt. Berlin, Sander, 1808 (neu hg. Hans-Michael Bock, Frankfurt a/M, 1979, p. 481-769, p. 754).

9 Lettre de Gentz à Adam Müller de mars 1808, in Briefwechsel zwischen Friedrich Gentz et Adam Heinrich Müller. 1800-1829, Stuttgart, 1857, p. 129.

10 Müller Adam, lors de recherches sur la noblesse de naissance de Fr. Buchholz, in Pallas, 1808, p. 83.

11 Heinz-Dieter Kittsteiner, Der Streit um Christian Jacob Kraus in den "Berliner Abendblättern" (2005), in http://www.textkritik.de/vigoni/kittsteiner1.htm [letzter Zugriff 24/03/2013], p. 29. 
conseiller à la cour danoise pour devenir écrivain à Berlin. Saul Ascher, qui en tant que juif n'était pas dans la fonction publique, échangea son existence de libraire contre une carrière de journaliste politique et travailla entre autres en tant que correspondant pour les Ecrits sur l'histoire de notre temps (Überlieferungen zur Geschichte unserer Zeit) d'Heinrich Zschokke. Karl Ludwig Woltmann, enfin, renonça à la perspective d'une chaire de professeur à Göttingen, car l'éditeur berlinois Unger lui fit une proposition plus avantageuse pour le périodique Histoire et Politique (Geschichte und Politik).

Ce type d'auteurs décrit ici, les écrivains de l'histoire politique, ne se limite pas à l'échelle régionale. On le trouve au contraire dans tous les territoires allemands et dans toutes les régions germanophones : dans la Confédération du Rhin (Johann Gottfried Pahl, Niklas Vogt, Peter Adolf Winkopp) ${ }^{12}$, en Alsace (Johann Friedrich Butenschön, Friedrich Christoph Cotta) ${ }^{13}$, dans le Wurtemberg (Ernst Ludwig Posselt) ${ }^{14}$, en Bavière (Joseph Widemann) ${ }^{15}$, en Autriche (Friedrich Gentz, Adam Müller, Friedrich Schlegel), en Saxe (Christian Daniel Voss) ${ }^{16}$, en Prusse (Friedrich Buchholz, Saul Ascher, Karl Ludwig Woltmann), en Suisse (Heinrich Zschokke, Paul Usteri), dans le Nord de l'Allemagne (August Hennings, Johann Wilhelm von Archenholz, Benedikt von Schirach) ${ }^{17}$ ou dans les pays baltes (Garlieb Merkel, Carl Gustav Jochmann) ${ }^{18}$.

12 Schuck Gerhard, Rheinbundpatriotismus und politische Öffentlichkeit zwischen AufklParung und Frühliberalismus. Kontinuitätsdenken und Diskontinuitätserfahrung in den Staatsrechtsund Verfassungsdebatten der Rheinbundpublizistik, Stuttgart, 1994 ; Fratzke-Weiss Birgit, Europäische und nationale Konzeptionen im Rheinbund. Politische Zeitschriften als Medien der politischen Öffentlichkeit, Frankfurt am M., Berlin u. Bern, 1997 ; Coignard Tristan, Vom „Reichspatriotismus"zum „Rheinbundpatriotismus"? Napoleons Reformkonzept und sein Widerhall im Umfeld des "Rheinischen Bundes“, in Marion George u. Andrea Rudolph (éd.), Napoleons langer Schatten über Europa, Dettelbach, 2008, p. 87-102.

13 Lachenicht Susanne, Information und Propaganda. Die Presse deutscher Jakobiner im Elsass (1791-1800), München, 2004, en particulier p $.459 \mathrm{ff}$.

14 Au sujet de Posselt : Salzbrunn, Ingeborg, Studien zum historischen Zeitschriftenwesen von der Göttinger Aufklärung bis zur Herausgabe der, Historischen Zeitschrift' (1859), thèse, Münster, 1968 ; Lang Hans-Joachim, Im Foyer der Revolution. Als Schiller in Tübingen Chefredakteur werden sollte: die Gründerzeit von Cottas „Allgemeiner Zeitung“, Tübingen, 1998, p. 77 ff.

15 Au sujet de Widemann : Piereth, Bayerns Pressepolitik und die Neuordnung Deutschlands nach den Befreiungskriegen, München, 1999 ; Fratzke-Weiss, Rheinbund, 1997 (voir réf. 12).

16 Au sujet de Voss : Rüdiger Axel, Staatslehre und Staatsbildung. Die Staatswissenschaft an der Universität Halle im 18. Jahrhundert, Tübingen, 2005.

$17 \mathrm{Au}$ sujet d'Archenholz : Ruof Friedrich, Johann Wilhelm von Archenholz. Ein deutscher Schriftsteller zur Zeit der französischen Revolution und Napoleons (1741-1812), Berlin, 1915 [photom. Repr. Vaduz, 1965] ; Rieger Ute, Johann Wilhelm von Archenholz. Eine historischanalytische Untersuchung zur Aufklärung in Deutschland, Berlin, 1994 ; au sujet de Schirach : Böning Holger, „Ein wahrer Philosophischer Royalist“. Gottlob Benedict von Schirach und seine Publizistische Tätigkeit, in Christoph Weiss u. Wolfgang Albrecht (éd.), Von, Obscuranten "Eudämonisten" Gegenaufklärerische, konservative und antirevolutionäre Publizisten im späten 18. Jahrhundert. St Ingbert, 1997, p. 403-444.

18 Au sujet de Merkel : Bosse Heinrich, Vom Schreiben leben. Garlieb Merkel als Zeitschriftsteller, in Otto Heinrich Elias (éd.), Zwischen Aufklärung und Biedermeier, Lüneburg, 2007. 


\section{Les réseaux européens}

Alors qu'un auteur comme Friedrich Gentz représente après le Congrès de Vienne, comme aucun autre, les réseaux de presse politique de la coalition antinapoléonienne et de la restauration, on peut étudier les réseaux de la presse politique pro-française et libérale à travers les exemples de Joseph Widemann et Friedrich Buchholz. Comme l'a montré Birgit Fratzke-Weiß, les périodiques politiques de langue allemande les plus importants défendaient dans l'ensemble un programme pro-napoléonien explicite : cela est valable pour les Annales européennes dans le sud-ouest de l'Allemagne, tout comme pour les Époques (Zeiten) en Allemagne centrale, la Minerva dans le Nord de l'Allemagne ou encore les journaux suisses d'Heinrich Zschokke, les Micelles pour les toutes nouvelles connaissances du monde (Miscellen für die Neueste Weltkunde) et les Écrits sur l'histoire de notre temps (Überlieferung zur Geschichte unserer Zeit) ${ }^{19}$.

En outre, ces réseaux de presse - à l'image d'autres réseaux de communication de l'époque napoléonienne, comme par exemple le réseau routier - se sont révélés plus stables que l'Empire lui-même et sont restés actifs au-delà de l'époque napoléonienne. Widemann comme Buchholz illustrent les perspectives d'ascension sociale dans le domaine journalistique et comptent parmi les agents les plus importants dans l'empire de la presse de Johann Friedrich Cotta. Tout comme Buchholz était l'un des journalistes les plus discutés en Prusse, Johann Friedrich Cotta (1764-1832) était l'éditeur le plus important, non seulement au sein de la Confédération du Rhin, mais aussi probablement à l'échelle de l'Allemagne. Des auteurs renommés comme Schiller et Goethe étaient employés sous contrat chez lui ; avec sa Gazette universelle (Allgemeine Zeitung), le Journal du matin pour les classes cultivées (Morgenblatt für die gebildeten Stände) et les Annales européennes, il avait construit un empire de la presse qui donnait le ton dans l'opinion publique ${ }^{20}$. Cotta employait des correspondants dans toutes les capitales européennes et dirigeait entre autres à Paris des filiales communes, des joint ventures, avec des maisons d'édition et des libraires installés sur place ${ }^{21}$. Dans une lettre à Cotta, Buchholz désigne d'ailleurs ce dernier d'une manière très révélatrice comme un "Napoléon parmi les libraires »"

19 Essentiel pour cet autre réseau européen de la presse politique de la Confédération du Rhin : Fratzke-Weiss, Rheinbund, 1997 ; Schuck, Rheinbundpatriotismus, 1994.

20 Fischer Bernhard, „Verlegerisches Know-how" im 18. und frühen 19. Jahrhundert: Die Verlagsstrategie Johann Friedrich Cottas, 1787-1795, in Schulz Günther (éd.), Geschäft mit Wort und Meinung. Medienunternehmer seit dem 18. Jahrhundert, München, 1999, p. 57-76; du même auteur : Von den „Europäischen Annalen“"zur Tribüne - J.F. Cottas politische Periodika. Universalhistorische Geschichtsschreibung und "Innere Staatsbildung", in Archiv für Geschichte des Buchwesens, 50 (1998), p. 295-315 ; Neugebauer-Wölk Monika, Revolution und Constitution: die Brüder Cotta; Eine biografische Studie zum Zeitalter der Französischen Revolution und des Vormärz, Berlin, 1989.

21 Il publia ainsi avec l'imprimeur Xhrouet à Paris un Almanach des Dames.

22 Buchholz à Cotta, 5 janvier 1807, lettre n 15, in Iwan-Michelangelo D’Aprile, Die Erfindung 
Les lettres de Buchholz à Cotta vont largement au-delà d'une relation d'affaires entre un auteur et un éditeur ${ }^{23}$. En plus des discussions habituelles sur les honoraires, les œuvres à remettre et les conseils au sujet d'auteurs éventuels pour les nombreux journaux, de Christian von Massenbach à Friedrich von Raumer, on y trouve des commentaires et analyses détaillés de la situation géopolitique, ainsi que des discussions révélatrices sur la relation entre auteur et éditeur et sur l'envergure des publications d'histoire contemporaine. Enfin, Cotta - tout comme Buchholz plus tard - travailla en étroite collaboration avec Hardenberg, et cela dès sa période à AnsbachBayreuth dans les années 1790, puis de nouveau et avec plus d'intensité lors de la création de la Confédération germanique. Cotta devra aussi plus tard son anoblissement à Hardenberg ${ }^{24}$. Pour Buchholz, Cotta était une sorte d'agent de liaison vers une autre Allemagne au-delà de la Prusse.

Buchholz livre des descriptions détaillées de la vie dans la capitale prussienne : de l'entrée des troupes napoléoniennes dans Berlin en octobre $1806^{25}$, au suicide d'Heinrich von Kleist ${ }^{26}$, sans oublier le quotidien dans un État en faillite. Il ne regrette aucunement la défaite du vieil État prussien, dont les causes seraient à chercher bien avant les batailles d'Iéna et d'Auerstedt. En tant qu'État dynastique, il est d'après lui trop étroitement lié à l'ancienne monarchie des Hohenzollern pour qu'un nouvel État puisse en naître. La Prusse lui apparaît comme un anachronisme qui « n'a plus rien à voir avec les rapports actuels des choses $»^{27}$. Le 28 mai 1808, il considère l'effondrement de la Prusse comme scellé : " malgré toute apparence, l'idée de la Prusse disparait du monde européen ; car cette idée est intimement liée à la dynastie des Hohenzollern qui se retire désormais de la scène, comme les Bourbons du trône d'Espagne. $»^{28}$ Une lettre du 19 janvier 1810 nous montre clairement à quel point Buchholz, en tant que libéral en Prusse, se sent comme un apatride politique : « (...) je dois vous avouer que tout va très mal pour nous. Nous nous battons de notre mieux contre notre faillite. On ne sait pas encore si elle va se produire. Il est difficile de rester un spectateur indifférent devant tant de

der Zeitgeschichte. Geschichtsschreibung und Journalismus zwischen Aufklärung und Vormärz, Berlin 2013 („Napoleon unter den Buchhändlern“).

23 Les réponses de Cotta à Buchholz, tout comme les œuvres posthumes de Buchholz, n’ont malheureusement pas été conservées.

24 Fischer, Cottas politische Periodika, 1998, p. $300 \mathrm{ff}$.

25 Buchholz à Cotta, 13 novembre 1806, lettre n 13 (voir ref. 22)

26 Buchholz à Cotta, 26 novembre 1811, lettre n 82 (voir ref. 22)

27 Buchholz à Cotta, 30 octobre 1809, lettre $n^{\circ} 62$ (voir ref. 22) (,nicht mehr in den gegenwärtigen Zusammenhange der Dinge gehört").

28 Buchholz à Cotta, 28 mai 1808, lettre n 42 (voir ref. 22) („Allem Ansehen nach verschwindet die Idee Preussen aus der europäischen Welt; denn diese Idee hängt aufs Innigste mit der Dynastie der Hohenzollern zusammen, welche ihre Rolle ebenso ausgespielt hat, als die Bourbonen auf dem spanischen Thron"). 
bêtises qui s'enchaînent les unes après les autres ; je me suis cependant fermement décidé à devenir indifférent à tout ce qui concerne ma patrie, jusqu’à ce que j'aperçoive l'esprit du monde arriver jusqu'à nous. La nuit de barbares dans laquelle nous nous trouvons est à peine éclairée de quelques rayons par la raison. Nous nous enfonçons sans cesse dans la féodalité, dont nous voulons sortir ; et toutes nos dispositions publiques sont ainsi faites que la domination de l'esprit féodal ne peut prendre fin. Parce que la libéralité n'est pas dans les cœurs, elle n'est pas non plus dans les esprits. $»^{29}$

Dès le début, Buchholz exprime dans ce contexte l'espoir que la Prusse puisse se fondre dans la Confédération du Rhin, qu'il considère comme le germe d'un nouvel État national allemand. Pendant presque toute la durée du gouvernement Hardenberg, la Confédération du Rhin lui apparait comme un modèle d'après lequel doivent s'orienter les réformes en Prusse. Le royaume de Westphalie par exemple représente pour Buchholz un État exemplaire, «un véritable ferment qui ne peut épuiser ses forces tant que tous les autres royaumes allemands n'ont pas adopté les mêmes lois (organiques et civiques). „»0

Tout comme Buchholz, Joseph Widemann (1778-1826) est un de ces auteurs de métier à partir duquel il est possible d'étudier les réseaux européens du journalisme politique de l'époque napoléonienne et au-delà. Widemann était autrichien et débuta sa carrière comme auteur de récits de voyage - deux récits de voyage sur l'Istrie et la Vénétie nous ont été transmis ${ }^{31}$. En 1805, il travaillait au sein de la rédaction du Journal de Vienne (Wiener Zeitung) lorsque celui-ci devint pro-français pendant la troisième guerre de coalition ${ }^{32}$. Parallèlement, il comptait déjà parmi les correspondants de la Gazette universelle (Allgemeine Zeitung) de Cotta depuis 1804. Au cours de l'année 1806, Widemann se rendit à Paris et y travailla pour la presse napoléonienne. Entre 1806 et 1807, il travailla aussi pour le bureau de l'intendant français à Berlin, Louis-

29 Buchholz à Cotta, 19 janvier 1810, lettre $n^{\circ} 65$ (voir ref. 22) : " (...) im Vertrauen muss ich Ihnen sagen, dass es in jeder Hinsicht sehr schlecht um uns steht. Wir kämpfen mit unserem Bankerot so gut wir können. Ob er ausbleiben wird, steht noch dahin. Schwer ist es, ein gleichgültiger Zuschauer von allen den Albernheiten zu bleiben, von welchen die eine die andere jagt; aber ich habe mir fest vorgenommen, mich in Beziehung auf mein Vaterland zu indifferenzieren, bis ich sehe, dass der Weltgeist wirklich über uns kommt. Die Nacht der Barbaren in welcher wir liegen, wird kaum durch einige Vernunftstrahlen aufgehellt. Wir plumpen immer wieder in die Feudalität zurück, aus welcher wir uns erheben wollen; $u$. alle unsere öffentlichen Maaßregeln sind von solcher Beschaffenheit, dass der Herrschaft des Feudal-Geistes kein Ende gemacht werden kann. Weil die Liberalität nicht im Gemüthe ist, so ist sie auch nicht im Geiste".

30 Buchholz à Cotta, 18 janvier 1811, lettre nº 76 (voir ref. 22) : "Ein wahrer Gährungsstoff, der seine Kraft nicht eher verlieren kann, als bis alle übrigen deutschen Königsreiche dieselben Gesetze (organische u. bürgerliche) angenommen haben".

31 Fratzke-Weiss, Rheinbund, 1997 ; Piereth, Bayerns Pressepolitik, 1999 ; Widemann, Streitzüge durch Innerösterreich, 1801 et Streitzüge an Istriens Küsten, 1810.

32 Piereth, Bayerns Pressepolitik, 1997, p. 156-161. 
Pierre Édouard, baron de Bignon ${ }^{33}$. Sa carrière croise ici le chemin d'HenriJacques-Guillaume Clarke : il fut tout d'abord traducteur chez lui, au Cabinet topographique. Lorsque Clarke devint le ministre de la guerre de Napoléon, Widemann fut également transféré à un poste au sein du département de cartographie du ministère de la guerre, où il travailla en tant que secrétaireinterprète. Il faisait en outre partie de la rédaction du Journal de l'Empire. De plus, Widemann était la figure centrale du réseau de correspondants de Cotta à Paris ${ }^{34}$. Il ne se contentait pas d'enrichir les Annales européennes avec des articles et des rapports de correspondants, mais établissait également pour Cotta les contacts avec les services administratifs du gouvernement français, les journalistes, éditeurs, imprimeurs ou graveurs et l'informait des nouvelles parutions intéressantes sur le marché du livre français ${ }^{35}$. Par l'intermédiaire de Cotta, Widemann avait aussi fait la connaissance de Friedrich Buchholz.

On peut en effet observer dans les publications de Widemann des références de plus en plus nombreuses aux analyses économiques et géopolitiques de Buchholz ${ }^{36}$. C'est le cas d'essais comme le Système commercial et Code de la mer des océanocrates ${ }^{37}$ ou Encore une question concernant le café et le sucre; réponse demandée aux princes seulement et non aux savants ${ }^{38}$. L'essai de Widemann sur les océanocrates - paru également comme brochure séparée en français sous le titre Les océanocrates et leurs partisans - se révèle être, dans de longs passages, une paraphrase de la description de la monarchie universelle mercantile anglaise de Buchholz ${ }^{39}$. De plus, Widemann s'efforça constamment de faire connaitre les œuvres de Buchholz en France. Il parla des travaux de ce dernier dans le Journal de l'Empire ou dans l'Esprit des Journaux ${ }^{40}$. La recension détaillée de la Galerie des personnalités prussiennes trahit clairement des connaissances d'initié. L'identité de l'auteur de l'ouvrage publié en Prusse en langue française et allemande, sous couvert d'anonymat, et camouflé en

33 Widemann, "Die Sybillinischen Blätter », in Neue Feuerbrände, vol. 4, 1808.

34 Fratzke-Weiss, Rheinbund, 1997, p. 109-113.

35 Ibid., p. 115 ; voir aussi la correspondance parisienne de Cotta, DLAM Cotta.

36 Voir chap. 5.2 de cet ouvrage.

37 Widemann Joseph Georg, Handels-System und See-Codex der Oceanokraten, in Europäische Annalen 3 (1813), p. $52 \mathrm{ff}$.

38 Widemann Joseph Georg, Noch eine Preisfrage über Kaffee und Zucker; aber nicht von Gelehrten, nur von Fürsten zu lösen, in Europäische Annalen 2 (1808), p. 209-215.

39 Widemann Joseph Georg, Les Océanocrates et leurs partisans ou la guerre avec la Russie en 1812 par M. Widemann (de Vienne en Autriche), Paris, 1812.

40 Widemann à Cotta, Paris, 29 juin 1811 : "Buchh. Werken, die ich im J. de l'E. anzeigen möchte, seh“ich mit Sehnsucht entgg ", DLAM CA Briefe J. Widemann, Nr.37 ; Widemann à Cotta, 28 septembre 1811 : «Von Buchholz erbitte ich mir nur eins der neuesten Werke zu schicken; für ihn und mich wäre es gleich zuträglich. » DLAM CA Briefe J. Widemann, Nr.42. La discussion au sujet de la Galerie Preußischer Charaktere se trouve dans L'esprit des journaux français et étrangers, vol. 5, 1808, p. 89-115 et vol. 6, p. 31-48. 
traduction du français, est ainsi clairement révélée - alors qu'en Prusse, on continue à débattre vivement sur l'identité de l'auteur ${ }^{41}$.

Widemann réussit même à faire connaître les travaux de Buchholz au sein des cercles les plus hauts placés du gouvernement français. Peu de temps après qu'il se fit envoyer par Cotta l'ouvrage Rom und London de Buchholz, afin de le remettre au ministre de la guerre Clarke, ce dernier fit un rapport à Napoléon, dans lequel il loue l'écrit de Buchholz comme "l'analyse la plus fine de la situation politique en Europe " et conseille la publication d'une traduction française. Le 30 décembre 1808, Clarke écrit à Napoléon : «Un Mr. Buchholz à Berlin m'a dernièrement adressé un ouvrage en allemand, intitulé Rome et Londres, ou de l'essence de la Monarchie universelle, qui contient peut-être tout ce qui s'est dit de plus sage, de mieux pensé et de plus approfondi sur l'état politique de l'Europe, sur le Système des Anglais, sur celui de la France à leur égard, et sur la manière dont les Gouvernements du Continent devraient envisager la lutte qui s'est établie avec l'Angleterre. $»^{42}$

Même si le jugement de Clarke sur l'essai de Buchholz est largement guidé par une stratégie de propagande, cette lettre démontre toutefois l'existence d'un réseau de presse à l'échelle européenne durant l'époque napoléonienne. Il était auparavant presque impensable que les analyses politiques d'un autodidacte issu d'un milieu pauvre de la province du Brandebourg puissent être lues jusque dans les sphères du gouvernement français grâce à l'intermédiaire d'un auteur de récits de voyage autrichien et d'un éditeur du Wurtemberg.

\section{Continuités}

Ces réseaux de presse furent plus stables que l'Empire napoléonien lui-même et perdurèrent même après l'effondrement de ce dernier. Durant la période de réforme en Allemagne, Buchholz et Widemann, les deux célèbres partisans de la France de l'époque napoléonienne, devinrent des employés de presse au service de l'État en Bavière et en Prusse. Après avoir perdu son travail à Paris, Widemann chercha de l'aide, en 1814, auprès de Cotta qui le fit venir à Stuttgart, où il continua de travailler pour les Annales européennes et le Journal du matin pour les classes cultivées (Morgenblatt für die gebildeten Stände). Lorsque Widemann fut chassé du Wurtemberg sous la pression de l'Autriche en décembre 1814, Cotta l'envoya à Augsburg où il était censé travailler pour la Gazette universelle (Allgemeine Zeitung). Mais dès le début de l'année 1815, Widemann fut appelé par le ministre bavarois Maximilian von Montgelas afin

41 L'ordre des portraits est chamboulé par rapport à l'original et l'autoportrait de Buchholz est placé au début.

42 Cité d'après Granier, Herman, Berichte aus der Franzosenzeit 1807-1809 [=Publikationen aus den Königlich Preussichen Staatsarchiven, 48. Band], Leipzig, 1913, p. 323. 
de transformer l'Allemannia en un journal officiel bavarois qui avait pour rôle de soutenir médiatiquement la politique de réforme. Widemann démissionna de son travail à la Gazette universelle de Cotta, car celui-ci n'était, d'une part, pas satisfaisant d'un point de vue financier, et d'autre part, parce qu'on lui offrait la possibilité de participer à la " grande réforme d'un journal »3.

Friedrich Buchholz fut contacté peu de temps après l'entrée en fonction d'Hardenberg en tant que chancelier de l'État au cours de l'hiver 1810-1811 au sujet d'un éventuel poste et travailla avec ce dernier jusqu'à sa mort en 1822. À partir de mars 1811, Buchholz reçut un salaire mensuel de 90 talers et devint une sorte de mandataire de la Confédération du Rhin ${ }^{44}$. Dans une lettre d'Hardenberg à Buchholz du 16 mars 1811, on trouve le passage suivant : Buchholz doit " en tant qu'homme qui possède des connaissances historiques et perçoit ce qui est important au moment présent ", évaluer quels sont " les aménagements internes des États voisins » que l'on pourrait adopter et ceux qu'il faut rejeter ${ }^{45}$. Suivant cette mission, Buchholz a accompagné les projets de réforme les plus importants, qu'il s'agisse de l'édit sur les finances, de l'édit d'émancipation des juifs ou de la question de la représentation nationale, avec ses commentaires publics ou ses études dans la presse politique. Buchholz conçut entre autre un aperçu comparatif des Constitutions de France, d'Italie et de Westphalie ${ }^{46}$ et fit d'après ces modèles des propositions de Réforme de l'Empire allemand en un État central ${ }^{47}$.

Le premier écrit publié par Buchholz, qui montre le début d'une coopération avec Hardenberg, s'intitule Considérations libres sur l'ordonnance du 27 octobre concernant les finances et est conçu comme une lettre ouverte adressée aux concitoyens ; il parut directement après la proclamation de l'édit sur les finances chez l'éditeur Matzdorff à Berlin avec l'autorisation personnelle d'Hardenberg. L'édit comprend la première d'une série de promesses constitutionnelles qu'Harden-

43 Piereth, Bayerns Pressepolitik, 1999, p. 174 f. („große(n) ZeitungsReformation“).

44 Le 14 juin 1816, les payes mensuelles d'Hardenberg sont transformées en la somme annuelle de 1080 talers.

45 Hardenberg à Buchholz, le 16 mars 1811, Geheimes Staatsarchiv Preussischer Kulturbesitz (GStA PK) I. HA, Rp. 74 N III Nr.40, Bl.1 f. : " Bei der täglich wachsenden Nothwendigkeit, von den innern Einrichtungen der benachbarten Staaten Kenntnis zu nehmen, damit man das Heilsame nachahme, das Verderbliche vermeide, hat es mir räthlich geschienen, durch einen Mann, der mit historischen Kenntnissen die Einsicht des für den jetztigen Moment Wichtigen verbindet die zu jedem Zweck erforderlichen Ausmittelungen vornehmen zu lassen (...). Sie werden zwar in der Regel die Gegenstände der Bearbeitung aus meinem Bureau vorgezeichnet erhalten; doch auch wird es mir sehr angenehm seyn, wenn Sie selbst bei Ihrer ausgebreiteten Kenntniss mich auf die Puncte aufmerksam machen, welche eine Beleuchtung und Darlegung verdienen, und welche in fremden Staaten bereits bearbeitet und ausgeführt sind (...)».

46 Buchholz, Verfassungen Frankreichs, Italiens und Westfalens, GStA PK VI. HA, Nl Albrecht, Nr.32

47 Buchholz, Umbildung des Deutschen Reiches zu einem zentralen Staat, GStA PK VI. HA, Nl Albrecht, Nr.30. 
berg extorque au roi. Buchholz conclut son essai en affirmant que l'édit sur les finances d'Hardenberg constitue le premier pas vers l'adoption de la constitution française par la Prusse et représente ainsi la condition pour l'instauration d'une paix durable en Europe. Il va même plus loin et suppose dans une louange à Frédéric-Guillaume III pleine de sous-entendus que cette évolution a toujours correspondu à ses vœux les plus secrets, qu'il n'avait cependant pas pu s'imposer face aux forces conservatrices et aux détenteurs de privilèges, jusqu'à ce que le vieil État de Prusse soit détruit " par un coup porté de l'extérieur » et que cette "égalité devant la loi ", que Buchholz présente comme l'exigence politique principale à l'ordre du jour, ait été rendue possible.

Même après l'époque napoléonienne, Buchholz tient fermement au rôle de modèle que représente la constitution française et fait entrer en ligne de compte le " miroir transrhénan " de la France comme antidote contre les tentatives de restauration des anciens ordres, comme le montre la lettre du 18 avril $1817:$ : Je considère le rétablissement d'une constitution qui distingue différents ordres comme l'absurdité la plus complète à laquelle on puisse se laisser aller. Cette organisation était adaptée pour le $\mathrm{XIV}^{\mathrm{e}}$ et $\mathrm{XV} \mathrm{V}^{\mathrm{e}}$ siècle, mais n'est pas valable pour le XIX ${ }^{\mathrm{e}}$. Si elle avait eu une valeur aussi décisive que certains le prétendent, elle ne se serait pas effondrée. À une époque où l'administration est aussi développée que la nôtre, la représentation doit prendre une autre forme quautrefois, et le pire des choix que l'on puisse faire à cet égard est de rétablir, ne serait-ce que de nom, la société d'ordres. Le miroir transrhénan donne à ce sujet suffisamment d'explications si on souhaite les trouver et s'en servir. Mais malheureusement, nous nous croyons désormais bien plus malins que les Français ${ }^{48}$."

Alors que le groupe de journalistes et d'historiens libéraux et favorables à un système constitutionnel en France se retrouve effectivement à des postes stratégiques du pouvoir avec la Révolution de Juillet, et que des intellectuels comme François Guizot, Adolphe Thiers, François-Auguste Mignet, Augustin Thierry ou Jules Michelet accèdent à des postes administratifs hauts placés ou même à des postes ministériels, aucune évolution de ce genre ne se produit en Allemagne. On y constate au contraire, en particulier dans les deux États les plus puissants, la Prusse et l'Autriche, dès 1819-1820, une fin des réformes et une politique de plus en plus réactionnaire. Des auteurs libéraux comme

48 Buchholz à Cotta, 18 avril 1817, lettre $n^{\circ} 89$ (voir ref. 22) ("Ich halte die Zurückführung einer sogenannten ständischen Verfassung für den größten Unsinn, dem man sich hingeben kann. Diese Form war gut für das vierzehnte u. fünfzehnte Jahrhundert; aber sie taugt nicht für das neunzehnte. Hätte sie einen so entschiedenen Werth gehabt, wie Einige wollen, so würde sie nicht untergegangen seyn. In einer Zeit, wo die Verwaltung so ausgebildet ist, wie in der unsrigen, muss die Repräsentation einen anderen Charakter haben, als ehemals, $u$. der erste von allen Misgriffen, die man in dieser Hinsicht begehen kann, ist, das ständische Wesen, wär es auch nur dem Namen nach, zurückzurufen. Der transrheinische Spiegel giebt hierüber Aufschlüsse genug, wenn man sie finden u. benutzen will. Doch unglücklicher Weise glauben wir jetzt weit klüger zu seyn, als die Franzosen. ») 
Buchholz souffrent d'une censure accrue et les nouveaux courants politiques des années 1830 comme le mouvement Jeune-Allemagne (Junges Deutschland) se forment principalement en exil - et en particulier à Paris, la destination privilégiée de ces exilés, notamment des journalistes politiques comme Ludwig Börne, Heinrich Heine ou Karl Marx.

\title{
Iwan-Michelangelo D'Aprile
}

Unité de Potsdam

daprile@uni-potsdam.de

\section{Résumé}

Traduit de l'allemand par Mathilde Lerenard

Les bouleversements politiques qui caractérisent la Révolution et l'ère napoléonienne vont de pair avec les transformations du marché de la presse, l'importance croissante de la sphère publique, ainsi qu'un intérêt grandissant pour l'historiographie contemporaine à la croisée entre analyse politique et historicisation du présent. C'est dans ce contexte que naît un auteur d'un nouveau genre dans tous les grands États européens que l'on peut qualifier d'" historien journaliste ». Dans cet article, ces évolutions sont présentées à travers l'exemple des États allemands. Les opportunités de carrière toujours plus nombreuses pour les intellectuels sont préalablement discutées : celles-ci se situent dans des sphères sociales encore non établies qui gravitent entre une politique énergique de l'État en matière de presse et le développement d'un marché de périodiques historiques. Les relations franco-allemandes dans le champ du journalisme historique sont ensuite présentées à partir des exemples de deux journalistes allemands très influents en leur temps (Joseph Widemann et Friedrich Buchholz). Enfin, la continuité de ces réseaux de presse européens au-delà de l'ère napoléonienne, lors de la période de réformes dans l'espace germanique, est démontrée.

\section{Mots-clefs}

Transformation de la sphère publique, réseaux de presse européens, histoire de l'historiographie, histoire contemporaine, politique en matière de presse.

\begin{abstract}
The political changes in the revolutionary and Napoleonic age went hand in hand with changes in the press market, a rising significance of the public sphere and a growing interest in immediate historiography at the interface of political analysis and the historicization of ongoing events. In the process of these developments a new type of author emerged in all the larger European states who can be called a "journalist historiographer" (" historien journaliste "). In this article, these developments are presented through the example of the German states. In a first step the growing career opportunities for intellectuals are discussed which are located in not-yet-established social spheres situated between the state's active press politics and a growing public market for historical periodicals. In a second step, the Franco-German relations in the field of historical journalism are sketched by referring to the examples of two of the most influential German journalists of that time (Joseph Widemann and Friedrich Buchholz). And finally, the continuities of these European press networks beyond the Napoleonic era in the German reform period are outlined.
\end{abstract}

\section{Keywords}

Change of the public sphere, European press networks, history of historiography, contemporary history, press politics. 